\title{
STRESS FIELDS IN PLANE IRROTATIONAL FLOWS OF VISCOUS AND PERFECTLY PLASTIC CONTINUA*
}

\author{
By \\ WAN-LEE YIN \\ Georgia Institute of Technology
}

\begin{abstract}
The viscous and viscoplastic stress fields in three families of plane irrotational flows are determined by using the properties of complex analytic functions. In these flows the slip line fields are defined by the continuation of an orthogonal pair of characteristics that are straight lines, logarithmic spirals or Cornu's spirals, respectively. The three families of solutions comprise all plane flows which, when subjected to changes in the time scale, continue to satisfy the dynamical equations of a viscoplastic continuum in the sense of Bingham.
\end{abstract}

1. Introduction. In a previous work [1], we obtained the class of all plane irrotational motions satisfying the dynamical equations of a rigid-perfectly plastic body when the body force is conservative or absent. Such plane motions are characterized by the property that the angles of inclination of their slip-lines fields - the Hencky-Prandtl nets-are plane harmonic functions. The motions in this class also possess the following physical significances:

(i) They are exact solutions of incompressible linearly viscous materials and of viscoplastic materials (in the sense of Bingham).

(ii) Under conservative body forces, these motions continue to satisfy the dynamical equations of viscous or viscoplastic bodies when they proceed at varied rates, i.e., when the time variable is monotonically reparameterized.

We remark that the irrotational property of the flows follows necessarily from condition (ii), according to a kinematical theorem valid for general incompressible bodies [2].

Let $u(x, y)$ and $v(x, y)$ denote the rectangular components of the velocity field. The complex velocities $w=u-i v$ of the flows in our class can be represented in one of the following ways:

$$
\begin{array}{ll}
\text { Family 1: } & w=C_{1} z+C_{2}, \\
\text { Family 2: } & w=C_{3} / z+C_{4}, \\
\text { Family 3: } & z=z_{0}+(C / m)^{1 / 2} \operatorname{erf} \zeta, \\
& w=w_{0}-i(m C)^{-1 / 2} \operatorname{erf}(i \zeta) .
\end{array}
$$

\footnotetext{
* Received January 29, 1981.
} 
Here $z=x+i y, \zeta$ is a complex parameter and the complex error function is defined by

$$
\operatorname{erf} \zeta=(4 / \pi)^{1 / 2} \int_{0}^{\zeta} \exp \left(-t^{2}\right) d t
$$

Also, $m$ is a real constant and $z_{0}, w_{0}, C$ and $C_{1}$ to $C_{4}$ are complex constants. Generally, these constants may all depend on time.

The first two families include certain familiar flows in hydrodynamics and viscous flow theory. The flows of Eq. (1) have constant velocity gradients. The velocity fields of Eq. (2) are obtained by superposing uniform flows over a flow with a source, a sink (of either the radial or spiral pattern) or an irrotational vortex at the origin. On the other hand, Family 3 consists entirely of new solutions of viscous and plastic bodies. The geometry of their velocity fields may be described by introducing an orthogonal net of parametric curves (defined by constant real or imaginary parts of the complex parameter $\zeta^{2}$ ), and the nature and positions of flow singularities and stagnation points may be determined [1].

In the present paper, the stress field in the three families of flows will be solved analytically for a linearly viscous or rigid-viscoplastic body. As is well known, in an incompressible body the deformation history and the constitutive equation determine the deviatoric stress but not the hydrostatic pressure. We first obtain the deviatoric stress in Sec. 2 and then determine the hydrostatic pressure in Sec. 3.

When the viscosity effect is absent, the results of our analysis belong to the welldeveloped plane-strain theory of rigid-perfectly plastic bodies. This theory uses characteristics and Riemann functions as standard tools of solution [3, pp. 349-367]. However, the present approach to irrotational flows and the corresponding stress fields is based mianly on the properties of analytic functions. Certain symmetry properties of the complex error function facilitate the representation and visualization of the flow and stress fields associated with Eq. (3). The orthogonal nets of slip lines obtained by this approach are then re-examined from the viewpoint of the characteristic initial-value problem for the slip line equation.

2. The deviatoric stress. Familiar flows of Families 1 and 2 will be considered first. The flow described by Eq. (1) has a constant stretching tensor. Hence for viscous and viscoplastic bodies the stress field is constant and the slip lines are orthogonal straight lines. The stretching tensor of the flow described by Eq. (2) has the following components in a polar coordinate system:

$$
\left[\begin{array}{ll}
D_{r r} & D_{r \theta} \\
D_{r \theta} & D_{\theta \theta}
\end{array}\right]=\frac{\left|C_{1}\right|}{r}\left[\begin{array}{rr}
-\cos \left(\arg C_{1}\right) & \sin \left(\arg C_{1}\right) \\
\sin \left(\arg C_{1}\right) & \cos \left(\arg C_{1}\right)
\end{array}\right] .
$$

Hence the polar components of the viscous stress are independent of $\theta$ and those of the plastic stress are independent of position. The principal stress trajectories (isostatics) intersect the radial lines and concentric circles at constant angles, $-\left(\arg C_{1}\right) / 2$ and $\left(\pi-\arg C_{1}\right) / 2$, respectively. They are orthogonal logarithmic spirals. The principal values of the viscous stress $\mathbf{S}_{v}=2 \mu \mathbf{D}$ are inversely proportional to the radial coordinate $r$, while those of the plastic stress $\mathbf{S}_{p}=(k / D) \mathbf{D}$ are of course independent of position. Here $k$ denotes the yield stress in pure shear and $\mu$ is the Newtonian viscosity. Since the slip line fields bisect the isostatics, their trajectories also form a net of orthogonal logarithmic spirals. This slip line pattern is known in the literature and is shown with the corresponding streamlines in Figure 16 of [3]. 
Next we consider the new flows described by Eq. (3). These flows are geometrically similar to a model flow defined by the parametric representation

$$
z(\zeta)=\operatorname{erf} \zeta, \quad i w(\zeta)=\operatorname{erf}(i \zeta)
$$

In fact, every flow described by Eq. (3) appears, at any instant of time and to an appropriately oriented observer with suitable units of length and time, as a uniform flow superposed on the model flow (5). Consequently, in the following analysis the stress field will first be obtained for this model flow.

Let $D$ and $\Psi$ be the modulus and argument, respectively, of the complex velocity gradient $g$. From Eqs. (4) and (5) we obtain

$$
g \equiv D e^{i \Psi}=\frac{d w}{d z}=\frac{d w}{d \zeta} / \frac{d z}{d \zeta}=e^{2 \zeta^{2}}=e^{2 \alpha}(\cos 2 \beta+i \sin 2 \beta) .
$$

Hence the deviatoric stress in a Bingham viscoplastic material has the components

$$
[\mathbf{S}]=\left(k+2 \mu e^{2 \alpha}\right)\left[\begin{array}{rr}
\cos 2 \beta & -\sin 2 \beta \\
-\sin 2 \beta & -\cos 2 \beta
\end{array}\right] .
$$

It follows that the principal values $\pm\left(k+2 \mu e^{2 \alpha}\right)$ depend only on the parameter $\alpha$, and the principal directions of stress $e^{-i \beta}$ and $e^{i(\pi / 2-\beta)}$ depend only on the other parameter $\beta$. This allows complete determination of the deviatoric stress field from the following orthogonal net of curves in the $z$-plane:

$$
\operatorname{Re} \zeta^{2}=\alpha=\text { constant, } \quad \operatorname{Im} \zeta^{2}=\beta=\text { constant. }
$$

According to Eq. (5a) the conformal mappings from the complex variables $\zeta$ and $\zeta^{2}$ to $z$ are defined by the inverse error function

$$
\begin{gathered}
\zeta \equiv \xi+i \eta=\text { inverf } z, \\
\zeta^{2} \equiv \alpha+i \beta=(\text { inverf } z)^{2} .
\end{gathered}
$$

The complex inverse error function has branch points of infinite order at $z= \pm 1$. This function is the analytical continuation of the real inverse error function (defined on the interval $-1<x<1$ ) continued around the two branch points in both the clockwise and the counterclockwise directions. The two families of orthogonal curves of Eq. (8) are illustrated in Fig. 1 for the principal branch of the $z$-plane. We call these curves $\alpha$-lines and $\beta$-lines respectively. The $\alpha$-lines defined by $\alpha=0$ is the well-known Cornu spiral [4].

In photoelasticity an isochromatic is a curve along which the principal stress difference has a constant value. An isocline is the locus of points with the same principal directions of stress. Since in the model flow the principal stresses depend only on $\alpha$ and the principal directions depend only on $\beta$, each $\alpha$-line is an isochromatic and each $\beta$-line is an isocline. From the family of isoclines ( $\beta$-lines) we may construct the isostatics and the slip lines by the usual graphical procedure. Since (4), (5), (9), and (10) yield

$$
d z=\sqrt{2 / \pi} e^{-\zeta^{2}} d \zeta, \quad \arg (d z)=-\beta+\arg (d \xi+i d \eta),
$$

it is clear that if $d \xi=0$ or $d \eta=0$ then $d z$ is parallel to a principal direction, $e^{i(\pi / 2-\beta)}$ or $e^{-i \beta}$. Hence the isostatics coincide with the curves $\xi=$ constant and $\eta=$ constant in the $z$-plane. 
The analytical expressions for the various families of curves may be summarized as follows

Isostatics: $\quad \operatorname{Re}($ inverf $z)=\xi=$ const,

$$
\operatorname{Im}(\text { inverf } z)=\eta=\text { const, }
$$

Isochromatics: $\operatorname{Re}\left\{(\text { inverf } z)^{2}\right\}=\alpha=\xi^{2}-\eta^{2}=$ const,

Isoclines: $\quad \operatorname{Im}\left\{(\text { inverf } z)^{2}\right\}=\beta=2 \xi \eta=$ const.

Along a $\beta$-line $(\beta=$ const $)$ Eq. (11b) reduces to

$$
\arg (d z)=-\beta-\arctan \left\{\sqrt{1+\left(\frac{\alpha}{\beta}\right)^{2}}-\frac{\alpha}{\beta}\right\}=-\beta-\frac{1}{2} \arctan (\beta / \alpha) .
$$

Hence the tangent vector of the $\beta$-line changes from $e^{-i(\pi / 4+\beta)}$ at its intersection with Cornu's spiral $(\alpha=0)$ to $e^{-i \beta}$ and $e^{-i(\pi / 2+\beta)}$, respectively, as the branch point $z=1$ $(\alpha=+\infty)$ and the point at infinity $(\alpha=-\infty)$ are approached. Hence one family of isostatics near the branch point have a radial pattern. At large distances away from the branch point, the tangents of $\alpha$ - and $\beta$-lines also approach the principal axes of stress. Along Cornu's spiral the tangential direction $e^{i(\pi / 4-\beta)}$ agrees with a slip line direction. Hence this curve is a slip line.

Geometrically, a Cornu spiral is characterized by the proportionality of the curvature and the arc length. In the main branch of the $z$-plane there are two Cornu spirals $\xi= \pm \eta$ intersecting orthogonally at the origin, where they have zero curvature. With these two curves as characteristics, the characteristic initial-value problem associated with the differential equation of slip line fields has a unique solution in terms of Riemann's function [3, $\mathrm{p}$. $361 ; 5$, p. 155]. Writing $=-\beta \pm 4 \pi$, the slip line field equation is

$$
(2 \sin 2 \theta)_{x y}+(\cos 2 \theta)_{x x}-(\cos 2 \theta)_{y y}=0 .
$$

This unique continuation from initial characteristics (Cornu's spirals) defines the slip line field in the model flow. Similarly, the system of orthogonal rectilinear slip lines of the flow (1) is the continuation from two perpendicular rectilinear slip lines, and the slip line field of the flow (2) is the continuation from two logarithmic spirals making constant angles arg $C_{1}$ and $\arg C_{1}-\pi / 2$ with the radial vectors. Thus, from the point of view of the characteristic initial-value problem for Eq. (13), the slip line fields in plane irrotational flows of a rigidperfectly plastic or viscoplastic body are defined by three types of initial characteristics: a pair of orthogonal straight lines, logarithmic spirals or Cornu's spirals.

As mentioned earlier, general flows in Family 3 are geometrically similar to the model flow (5), except possibly for differences in the length and time scales and the orientation of the coordinate axes. At each instant, the slip line field of a flow defined by Eq. (3) has a pattern geometrically similar to that obtained in Fig. 1, but the origin of the $\alpha-\beta$ net and the branch point are located at the points $z_{0}$ and $z_{0}+(C / m)^{1 / 2}$, respectively. The shearing stress in the slip direction has the value $k+\left(2 \mu e^{2 \alpha} /|C|\right)$.

3. The mean pressure. Having obtained the deviatoric stress $\mathbf{S}$, we next consider the mean stress $-p$. This may be obtained by integrating the momentum equation

$$
\rho \dot{\mathbf{v}}=-\operatorname{grad} p+\operatorname{div} \mathbf{S}+\rho \mathbf{b} .
$$


Let $\Phi$ be the velocity potential and $\chi$ be the potential of the body force $b$. Then we have

$$
p=\rho\left(\chi-\frac{\partial \Phi}{\partial t}-\frac{1}{2}|w|^{2}\right)+q,
$$

where $q$ is that part of the pressure needed to balance the deviatoric stress:

$$
\operatorname{grad} q=\operatorname{div} \mathbf{S}=\operatorname{div} \mathbf{S}_{p}=k \operatorname{div}(\mathbf{D} / D) .
$$

For the model flow we have

$$
\begin{gathered}
\frac{d \zeta^{2}}{d z}=\frac{d}{d z}(\alpha+i \beta)=\beta_{y}+i \beta_{x}, \\
\frac{d z}{d\left(\zeta^{2}\right)}=\frac{d}{d\left(\zeta^{2}\right)}(x+i y)=x_{\alpha}+i y_{\alpha}=y_{\beta}-i x_{\beta},
\end{gathered}
$$

where the subscripts indicate partial derivatives. Since the viscous stress is selfequilibrating, Eqs. (7) and (15) yield

$$
\begin{aligned}
& q_{x}=k\left[(\cos 2 \beta)_{x}-(\sin 2 \beta)_{y}\right]=-2 k\left[(\sin 2 \beta) \beta_{x}+(\cos 2 \beta) \beta_{y}\right]=-2 k \operatorname{Re}\left(e^{-i 2 \beta} \frac{d \zeta^{2}}{d z}\right), \\
& q_{y}=-2 k \operatorname{Im}\left(e^{-i 2 \beta} \frac{d \zeta^{2}}{d z}\right) \\
& q_{\alpha}=q_{x} x_{\alpha}+q_{y} y_{\alpha}=-2 k \operatorname{Re}\left[e^{-i 2 \beta} \frac{d \zeta^{2}}{d z} \overline{\left(\frac{d z}{d(\zeta)^{2}}\right)}\right] \\
& q_{\beta}=q_{x} x_{\beta}+q_{y} y_{\beta}=-2 k \operatorname{Im}\left[e^{-i 2 \beta} \frac{d \zeta^{2}}{d z} \overline{\left(\frac{d z}{d(\zeta)^{2}}\right)}\right] .
\end{aligned}
$$

From Eq. (11b) we obtain

$$
\arg \frac{d z}{d\left(\zeta^{2}\right)}=-\beta-\frac{1}{2} \arctan (\beta / \alpha),
$$

so that

$$
\begin{aligned}
e^{-i 2 \beta} \frac{d \zeta^{2}}{d z} \overline{\left(\frac{d z}{d(\zeta)^{2}}\right)} & =\exp (-i 2 \beta) \exp \left[-i 2 \arg \left(\frac{d z}{d(\zeta)^{2}}\right)\right] \\
& =\exp (-i 2 \beta) \exp \left[i\left(2 \beta+\arctan \frac{\beta}{\alpha}\right)\right]=\exp \left(i \arctan \frac{\beta}{\alpha}\right) \\
q_{\alpha} & =-2 k \cos \left(\arctan \frac{\beta}{\alpha}\right)=-2 k \alpha\left(\alpha^{2}+\beta_{2}\right)^{-1 / 2} \\
q_{\beta} & =-2 k \sin \left(\arctan \frac{\beta}{\alpha}\right)=-2 k \beta\left(\alpha^{2}+\beta^{2}\right)^{-1 / 2}
\end{aligned}
$$

Integrating and substituting the result

$$
q=-2 k\left(\alpha^{2}+\beta^{2}\right)^{1 / 2}
$$


into (14), we obtain

$$
p=\rho\left(\chi-\frac{\partial \Phi}{\partial t}-\frac{1}{2}|w|^{2}\right)-2 k\left(\alpha^{2}+\beta^{2}\right)^{1 / 2} .
$$

For the special case of steady flow under vanishing body force the term $\rho(\chi-(\partial \Phi / \partial t))$ reduces to a constant. Furthermore, according to the following symmetry properties of the complex error function

$$
\operatorname{erf} \zeta=-\operatorname{erf}(-\zeta)=\overline{\operatorname{erf}(\bar{\zeta})}
$$

the velocity field $w$ of the model flow satisfies

$$
\overline{w(\xi+i \eta)}=i \overline{\operatorname{erf}(i(\xi+i \eta))}=-i \operatorname{erf}(\eta+i \xi) .
$$

Any pair of points in the $z$-plane of the form

$$
z=\operatorname{erf}(\xi+i \eta) \quad \text { and } \quad z^{*}=\operatorname{erf}(\eta+i \xi)
$$

can be located in Fig. 1 by starting from the Cornu spiral and following a $\beta$-line $(\beta=2 \xi \eta)$ in the two opposite directions until the tangent vector of the $\beta$-line has turned, respectively, through equal but opposite angles. Eq. (17) implies that

$$
\overline{w(z)}=-i z^{*} \text {. }
$$

Consequently,

$$
|\operatorname{grad} \Phi|^{2}=|w(z)|^{2}=\left|z^{*}\right|^{2}
$$

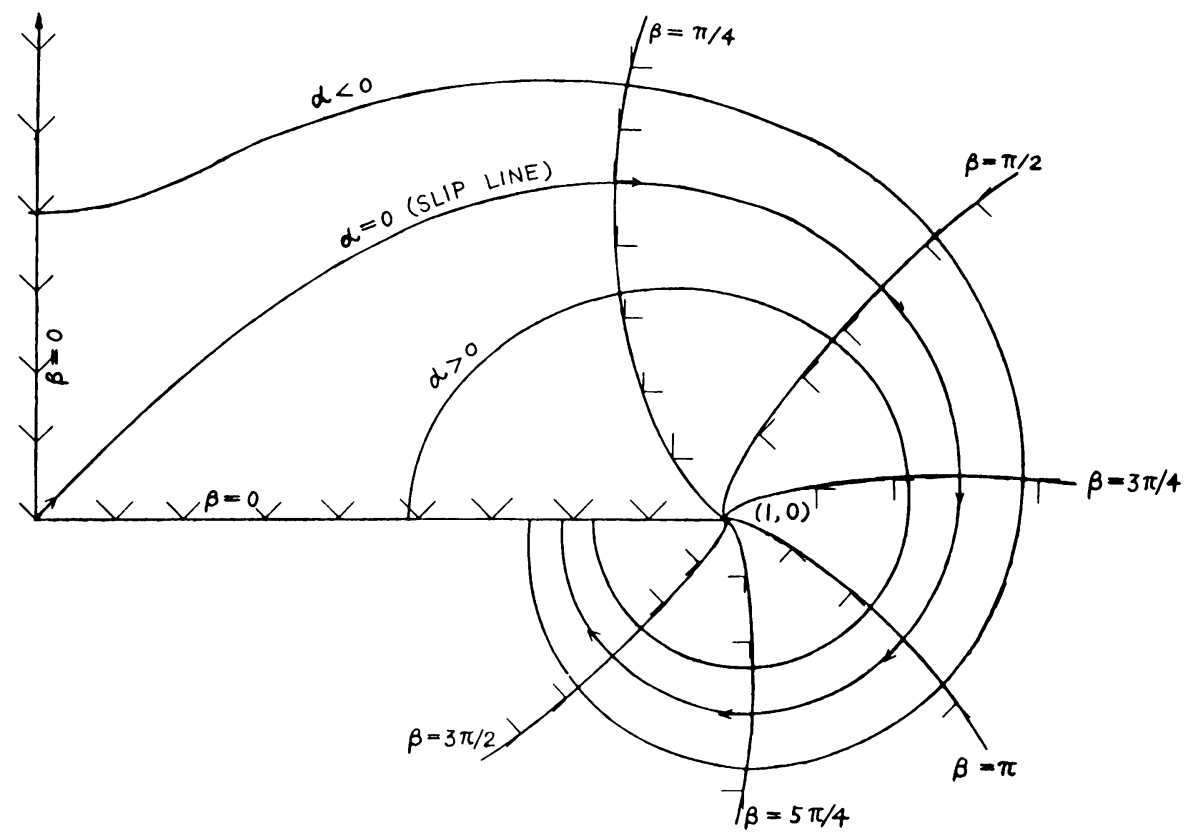

Fig. 1. $\alpha-\beta$ net and the slip line fields of flow (5). The fields remain constant along each curve $\beta=$ constant and are tangential or normal to the curve $\alpha=0$ (Cornu's spiral). 
and for a steady flow under vanishing body force Eq. (16) yields

$$
p=-\rho\left|z^{*}\right|^{2} / 2-2 k\left(\alpha^{2}+\beta^{2}\right)^{1 / 2}+p_{0},
$$

where $p_{0}$ is the pressure at the origin of the main branch of the $z$-plane (i.e. at $\alpha=\beta=0$ ). This expression allows the pressure field $p$ in the successive branches of the analytical continuation of the model flow to be completely known from the orthogonal net of $\alpha$ - and $\beta$-lines (shown in Fig. 1).

Eq. (18) also implies that $z^{*}=\operatorname{erf}\left(\eta_{n}+i \xi_{n}\right)=0$ at a stagnation point $z_{n}=\operatorname{erf}\left(\zeta_{n}+i \eta_{n}\right)$. Since the zeros of the complex error function are tabulated [6], the position of stagnation points can be obtained from the table of complex error function. At each stagnation point $z_{n}$ of the successive branches of flow continuation the pressure in the model flow is given by

$$
p=-2 k\left(\alpha_{n}^{2}+\beta_{n}^{2}\right)^{1 / 2}+\rho \chi,
$$

and the principal directions of stress are

$$
\exp \left(-i \beta_{n}\right), \quad \exp \left[i\left(\pi / 2-\beta_{n}\right)\right],
$$

which agree with the tangential directions of the orthogonal streamlines at the stagnation point.

Although Eq. (16) was derived for the model flow (5), it also yields the correct mean pressure for general flows of Eq. (3) provided that the parameters $\alpha$ and $\beta$ are, respectively, identified with the real and imaginary parts of $\left\{\text { inverf }\left[(m / C)^{1 / 2}\left(z-z_{0}\right)\right]\right\}^{2}$.

Finally, we have $q=0$ for the flows of Family 1 and

$$
q=-\frac{k}{\left|C_{3}\right|} \operatorname{Re}\left(C_{3} \log z\right)
$$

for Family-2 flows defined by Eq. (2). By substituting into Eq. (14), the pressure $p$ in steady flow is easily obtained.

\section{REFERENCES}

[1] D. R. Owens and W.-L. Yin, A class of exact solutions of the dynamical equations for rigid-viscoplastic bodies, Rheol. Acta 16, 223-226(1977)

[2] D. R. Owen and W.-L. Yin, On the possibility of detecting invariance of material response to changes in time scale, ZAMP 26, 605-610(1975)

[3] A. M. Freudenthal and G. Geiringer, The mathematical theories of the inelastic continuum, in Encyclopedia of physics, vol. VI, Elasticity and plasticity, edited by S. Flügge, Springer-Verlag, Berlin (1958)

[4] E. Jahnke and F. Emde, Tables of functions with formulae and curves, 4th Ed., Dover Publications, New York (1945), pp. 36-38

[5] R. Hill, Mathematical theory of plasticity, Clarendon Press, Oxford (1950)

[6] H. E. Salzer, Complex zeros of the error function, J. Franklin Inst. 260, 209-211 (1955) 